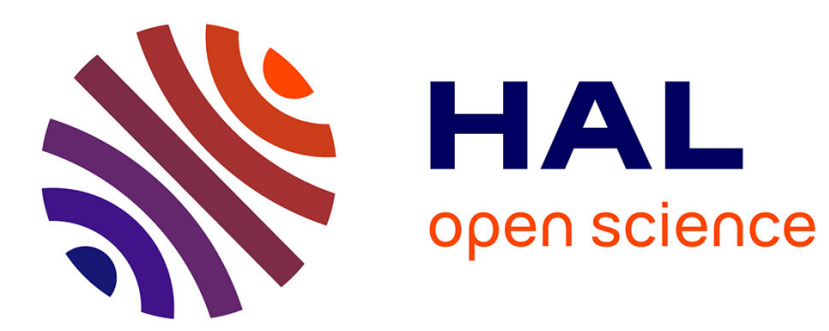

\title{
A holonic manufacturing approach applied to communicate concrete: Concept and first development
}

\author{
Hang Wan, Michael David, William Derigent
}

\section{To cite this version:}

Hang Wan, Michael David, William Derigent. A holonic manufacturing approach applied to communicate concrete: Concept and first development. 9th Workshop on Service Oriented, Holonic and Multi-agent Manufacturing, SOHOMA'19, Oct 2019, Valence, Spain. pp.414-425, 10.1007/978-3-03027477-1_32.hal-02421219

\section{HAL Id: hal-02421219 \\ https://hal.science/hal-02421219}

Submitted on 10 Jan 2020

HAL is a multi-disciplinary open access archive for the deposit and dissemination of scientific research documents, whether they are published or not. The documents may come from teaching and research institutions in France or abroad, or from public or private research centers.
L'archive ouverte pluridisciplinaire HAL, est destinée au dépôt et à la diffusion de documents scientifiques de niveau recherche, publiés ou non, émanant des établissements d'enseignement et de recherche français ou étrangers, des laboratoires publics ou privés. 
archives-ouvertes

\title{
A Holonic Manufacturing Approach Applied to Communicate Concrete: Concept and First Development
}

\author{
Hang Wan, Michael David, William Derigent
}

\section{To cite this version:}

Hang Wan, Michael David, William Derigent. A Holonic Manufacturing Approach Applied to Communicate Concrete: Concept and First Development. 9th Workshop on Service Oriented, Holonic and Multi-agent Manufacturing Systems for Industry of the Future, Oct 2019, Valence, Spain. pp.414-425, 10.1007/978-3-030-27477-1_32. hal-02421219

\section{HAL Id: hal-02421219 \\ https://hal.archives-ouvertes.fr/hal-02421219}

Submitted on 10 Jan 2020

HAL is a multi-disciplinary open access archive for the deposit and dissemination of scientific research documents, whether they are published or not. The documents may come from teaching and research institutions in France or abroad, or from public or private research centers.
L'archive ouverte pluridisciplinaire HAL, est destinée au dépôt et à la diffusion de documents scientifiques de niveau recherche, publiés ou non, émanant des établissements d'enseignement et de recherche français ou étrangers, des laboratoires publics ou privés. 


\title{
A Holonic Manufacturing Approach applied to communicate concrete: concept and first development
}

\author{
Hang WAN ${ }^{1}$, Michael DAVID ${ }^{1}$, William DERIGENT ${ }^{1}$ \\ ${ }^{1}$ Université de Lorraine, CRAN, UMR 7039, 2 avenue de la forêt de haye, \\ Vandoeuvre-lès-Nancy, 54516 Cedex, France \\ \{firstname. lastname\} @univ-lorraine.fr
}

\begin{abstract}
The rapid development of Internet of Things has enabled intelligent products that can be applied to the Industrial 4.0, Smart City, Smart Supply Chain and Smart Buildings. Its autonomy and flexibility make possible to construct intelligent manufacturing systems. In this paper, we present the McBIM project that is based on the concept of communicating materials. A holonic manufacturing approach is proposed to handle these problems. It aims to include a WSN to the product to make it more sensitive and aware of its own internal conditions. In our approach, the "sensing device" is an embedded Wireless Sensor Network that can collect "internal" data of the intelligent material. In this context, Physical and digital parts are defined and related to four main challenges. Some existing solutions of data collection architectures are discussed for the physical part. Furthermore, these algorithms are compared with the required performance metrics of our application. Then we conclude with some perspectives for future work.
\end{abstract}

Keywords: Internet of Things, holonic manufacturing approach, wireless sensor networks, communicating material, data aggregation, energy efficiency

\section{Introduction}

The Internet of Things (IoT) is widely used in manufacturing, monitoring applications and logistics, etc. The application of IoT makes the product more intelligent, its high flexibility brings new challenges to the Intelligent Manufacturing System (IMS): more flexible adaptability, higher computing capacity and control capabilities. In 2009, the Research Center for Automatic Control (CRAN) began to study the "communicating materials" concept, it is the material that can process, store data and communicate with the environment. With further study of Kubler [1] and Mekki [2], this concept has been applied to construction industry. The McBIM Project [3] (Material communicating with the BIM - Building Information Modelling) aims to design a "communicating concrete". Namely, it is the concrete equipped with embedded wireless micro-sensor network (WSN), which can measure the physical environment, store the information and exchange data with BIM platforms. Another objective is to demonstrate the usefulness of this new concept for different phases of a building's lifecycle, i.e. the manufacturing, construction and exploitation phases. 
The next section introduces the McBIM project and the concept of communicating concretes, details its main constraints and behaviors for each phase of its lifecycle. Section 3 presents a holonic manufacturing approach applied to the McBIM project and presents the composition of the holon by detailing its physical and digital parts, functionalities and the related challenges. Section 4 then focuses on the physical part and introduces some existing solutions of data collection architectures. A synthesis of those solutions and their use in our application case then are discussed in section 5. Last section concludes and gives some perspectives for the development of this work.

\section{Description of the McBIM project}

The McBIM project is funded by the French National Research Agency, and is coordinated by the CRAN with two other French laboratories (LAAS, LIB) and one company (360 SmartConnect/FINAO SAS). In this project, different partners focus on different areas. Where the CRAN works on the network and information management, the LAAS designs the sensing and communicating nodes, the LIB studies data interoperability and all these works are then implemented by 360 SmartConnect/FINAO SAS.

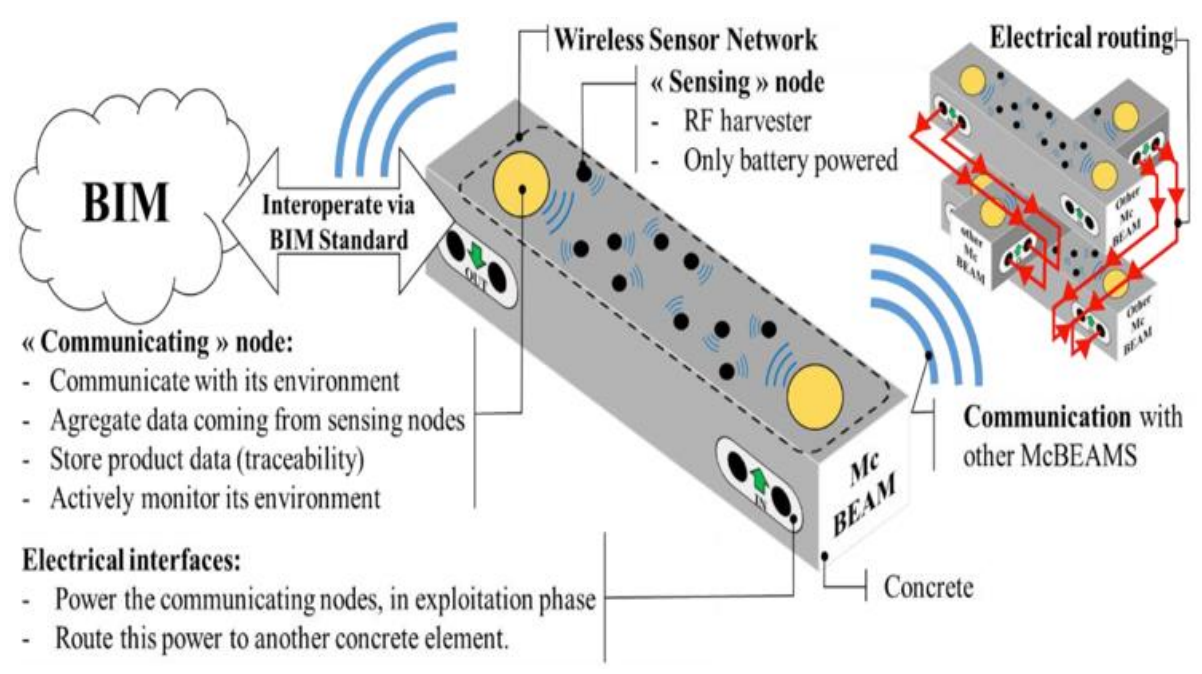

Fig. 1. The ANR McBIM project

The communicating concrete (see Fig. 1) consists of many sensing and communicating nodes. The sensing nodes will periodically monitor the physical parameters (like temperature, humidity ...) of the concrete. Communicating nodes aggregate received data and transmit it to monitoring center thanks to BIM standard. Besides, manufacturing data (like its physical properties or manufacturing actors) may be stored locally. The main constraint is that the concrete element has to live several decades from the manufacturing phase to the latest of the exploitation phase. 
Besides, the communicating concretes behaviors are different along its lifecycle (see Fig. 2). During the manufacturing phase, the WSN nodes are inserted and initialized. The communicating concretes periodically (by example every hour) monitor its physical status, store the physical propriety information, as well as the information of manufacturer, constructors and operators. These data are accessible directly via a reader device or remotely via the internet. For the construction phase, communicating concretes will be assembled together. In this case, auto-organization is then needed to dynamically define a 3D network to achieve energy savings. Due to the high flexibility in this step, concrete has to report its status frequently (such as every half-hour) to ensure the safety of construction and update the network information. When the construction is completed, the large 3D static WSN will regularly (by example every half-day) monitor struct health data (such as cracks, temperature, corrosion, etc.) to ensure the safety of the building.

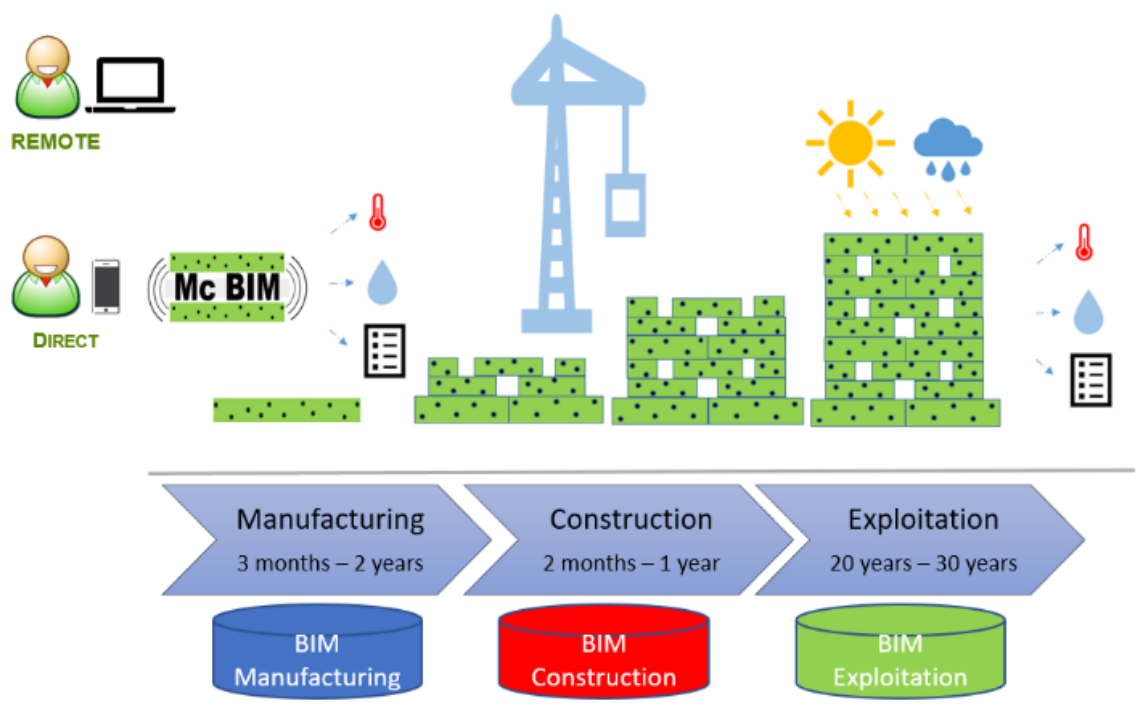

Fig. 2. McBIM communicating concrete lifecycle

\section{Holonic manufacturing approach of the McBIM project}

During the communicating concrete lifetime, more and more elements will work together, the data management and auto-organization of network will become complicated. In order to meet the changes of concretes over its lifecycle and ensure a longterm service, we propose a holonic manufacturing approach and outline its main challenges in Fig. 3. As described in [4], holonic manufacturing is a highly distributed control paradigm which is based on the concept of autonomous co-operating agents, called holons. A holon often consists of a physical processing part and an information 
processing part, it can work alone or co-operate with other holons. Moreover, a holon can also be part of another holon.

In our design, a McBIM holon consists of the real concrete element plus its WSN as physical part, and its dedicated agent as digital part. For example, the holon A (see Fig. 3) is composed of concrete element A and its agent A. The physical part monitors physical parameters and transmits to its dedicated agent. Subsequently, those data are processed at its agent in digital part. Each McBIM holon can work alone, it can also be assembled with another McBIM holon to construct higher holons as mentioned above. Indeed, in the physical part, the auto-organization of WSN makes the aggregation of the McBIM element A and B possible, they can then be viewed as a whole element $\mathrm{C}$. At this moment, we can also consider that holon $\mathrm{C}$ is made of the holon $\mathrm{A}$ and holon $B$. In order to realize this holonic manufacturing design, there are four main challenges as shown in Fig. 3: two in the physical part (1 and 3: the blue and green block), and two in the digital part ( 2 and 4: the red and yellow block).

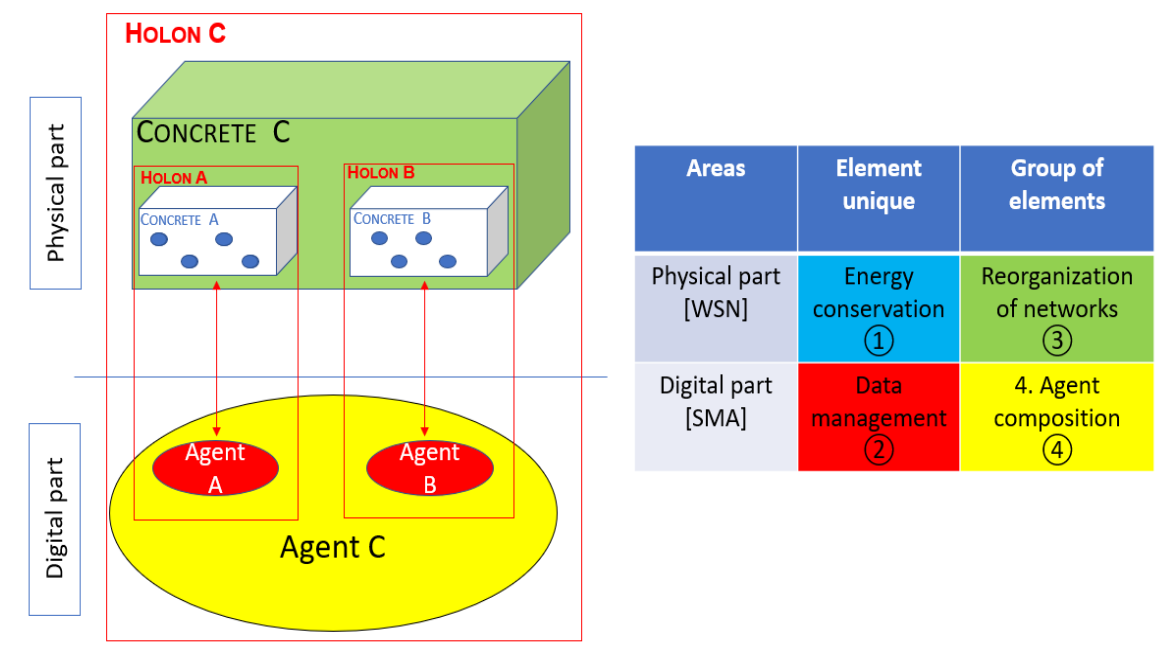

Fig. 3. Digital design and challenges

The first challenge (the blue block) is to ensure the long-term service of the communicating concrete, namely, the energy conservation in WSN within the concrete. Both the concepts of sensing and communicating nodes, the selection of different sensors, and its data collection technique should be taken into account. The second (the red block) is the realization of the link between the communicating concretes with its dedicated agents, and the data management at these agents. Both the data representation of physical part processing, and the data management for digital part need to be specified. The third challenge (the green block) is the auto-organization of network between different concrete elements in the physical part. At the end, the last is the specification of the hierarchical and heterarchical relationships between the dedicated agents. In this 
paper, we focus on the related research for the challenges in physical part: minimizing the energy consumption of data collection in WSN along its whole lifecycle (challenge 1 and 3$)$.

Aggregation techniques are widely used for data collection in WSN, as described in [5]. It needs three elements to be effective: an adapted structure of the network (for routing the data), aggregation functions (for fusing the data) and the data representation (for exploiting the data). Second and third elements are problems mainly depending on the application case. "Aggregation functions" depends on the service which is offered by the WSN and "Data representation" consists of the final exploitation of data by user (can be considered as an external problem for the WSN). In this work, we just focus on the preliminary step for aggregation: the WSN structure.

\section{$4 \quad$ Facing physical part challenges: WSN architecture}

In order to ensure a long-time service, we look for energy efficiency solutions for WSN data collection. To do this, several strategies exist, and among them, we focus on data aggregation techniques to minimize the number of messages. There are three main data aggregation architectures (chain-based, cluster-based and tree-based) detailed hereafter.

\subsection{Chain-based architecture}

A chain-based architecture is a WSN architecture where nodes communicate via a line structure. To construct this structure, several routing protocols have been proposed and are detailed as follows.

PEGASIS (Power-Efficient gathering in sensor information systems) [6] is the first chain-based routing protocol for WSN. As illustrated in Fig. 4, each node only communicates with its closest neighbors, and takes turn transmitting data to the BS (Base Station). In this case, energy consumption of transmission per round is reduced and network lifetime is increased with a random selection mechanism of the leader (c2 was selected as the leader for this round).

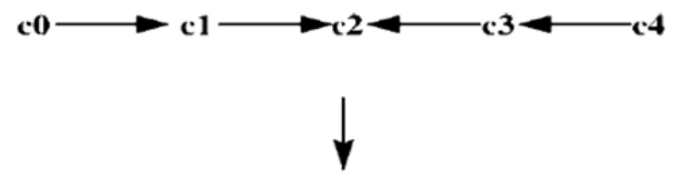

BS

Fig. 4. Data collection in PEGASIS 
In this approach, all nodes have global knowledge of the network and employ the same greedy algorithm. Each node can directly transmit to the BS. The computation of chain can be done at the base station, it then broadcasts the chain information to nodes. Or it can be constructed in a dynamic manner by all the sensor nodes. The dynamic construction begins with the furthest node. Each node performs the greedy algorithm to select its closest unvisited neighbor as next. In addition, they take turns to act as the leader with the probability: $i \bmod N$ where $i$ is the node number and $N$ is the number of nodes. The chain will be reconstructed if a node dies.

Since data fusion is performed at nodes, PEGASIS reduces the energy consumption in transmission for each round. Moreover, the random leader selection mechanism balances the consumption for the whole network and improves the lifetime. It is hard to ensure all nodes can connect with the BS in large WSNs. A long chain may have an important impact on the delay of transmission. In addition, the greedy algorithm in this protocol only considers the distance between nodes. The remaining energy at each node and the delay of transmission should be taken in account.

Based on PEGASIS, many improved algorithms have been proposed. A large part of these algorithms are related to the chain-cluster based approach, such as CCM (Chain-Cluster based Mixed routing) [7], CRBCC (Chain routing based on coordinatesoriented cluster) [8]. This kind of protocol combines the advantages of the chain-based architecture and the cluster-based. At beginning, it divides the networks into clusters. In each cluster, nodes form into one chain, a node will be selected as the chain leader. Between the different clusters, the selected cluster leaders then form a high-level chain, and one of them is selected to transmit information to the BS.

The differences between those algorithms are usually in the way of partitioning clusters, and the election of chain leader. Such in CCM, the reference network is an evenly distributed two-dimension network, each node with coordinate $(x, y)$. The partition of cluster only depends on the $x$ coordinate. The nodes $S(i, j)$ will be the leaders for the $j^{\text {th }}$ round, where $i, j$ are the horizontal and vertical coordinates. However, the partition of network in CRBCC depends on the $y$ coordinate and the numbers of nodes. Instead of using the greedy algorithm, CRBCC uses Simulated Annealing (SA) algorithm for forming both the low-level chain (into a cluster) and the high-level chain (between clusters). SA is used to calculate the lowest energy consumption route. This kind of chaincluster approach avoids the long chain disadvantage and reduces the delay. However, it increases the clustering overhead (i.e. the number of messages exchanged to construct the clustering), and the reconstruction of network is more complex.

Another kind of approach is the sub-network chain-based routing protocol. A Balanced Chain-Based Routing Protocol (BCBRP) has been proposed in [9]. The idea behinds this protocol is that it divides the network into different areas to reduce the energy consumption in maintaining of the WSN. The chain construction in BCBRP has four phases. First, the network is divided into different equal parts. Second, the closest nodes to the boundary are elected as bridge nodes which connect with the others sub- 
networks. Third, an algorithm that is similar to minimum spanning tree is used to construct the shortest chain in each part. Contrary to the greedy algorithm, the minimum spanning tree algorithm gives a global decision in order to connect all the nodes together with the minimum total route weight. Fourth, the chain leader is randomly selected from the area which has the largest number of nodes.

\subsection{Cluster-based architecture}

In a large WSN, the drawbacks of single chain approaches are obvious. Not only in term of delays, but also the maintaining of network structure. In the literature, another structure is proposed: the cluster-based approach where nodes are regrouped into clusters. Within a cluster, a node is elected as the cluster head $(\mathrm{CH})$ which receives information from its cluster members, aggregates and sends the aggregated data to the sink.

LEACH (Low Energy Adaptive Clustering Hierarchy) is the first proposed clusterbased protocol [10]. It is a distributed and self-organizing clustering protocol. A random mechanism is used to rotate $\mathrm{CHs}$ in order to balance the energy consumption. LEACH works with two phases for a round: setup phase and steady state phase. During the setup phase, the nodes organize themselves into clusters. A distributed probabilistic approach is used to elect the cluster leader. The threshold $\mathrm{P}_{\mathrm{i}}(\mathrm{t})$ is defined as (1):

$$
P_{i}(t)=\frac{k}{N-k *(r \bmod (N / k))} * c_{i}(t)
$$

Where $k$ is a predetermined cluster number, $N$ is the total number of nodes, $r$ is the current round number, $i$ is the node id and $c_{i}(t)$ is a function which indicates if the node has already been a $\mathrm{CH}$ in a previous round. For each round, sensor nodes randomly pick a number from 0 to 1 , and then compare with the threshold. One node becomes a leader if its picked number is bigger than the threshold. This probabilistic approach balances the energy at each node. When a $\mathrm{CH}$ is elected, it broadcasts a message to announce that it is the cluster leader. Surrounding nodes then decide which cluster to join based on the signal strength of the received messages.

The second phase is the data collection as shown in Fig. 5. All non-headers transmit data to their $\mathrm{CH}$. The leaders then transmit data directly to the sink after receiving all data. This algorithm improves the lifetime of network, but the single-hop routing used in this approach is inefficient for a large network. The leader consumes a large amount of energy when it is far from the sink. In addition, aggregation function could be implemented at the cluster-heads to reduce the transmission data.

Unlike the random election of leader in LEACH, Hybrid Energy-Efficient Distributed Clustering (HEED) [11] takes the residual energy and communication cost into account. An Average Minimum Reachability Power (AMRP) is defined for the estimation of communication cost. AMRP is the average of minimum power levels required for the non-leader nodes to connect with its $\mathrm{CH}$. For each round in HEED, each node computes its probability and becomes the leader if its value reaches 1 . Since the leaders are elected, the he surrounding nodes then choose a $\mathrm{CH}$ with the lowest AMRP cost. This residual 


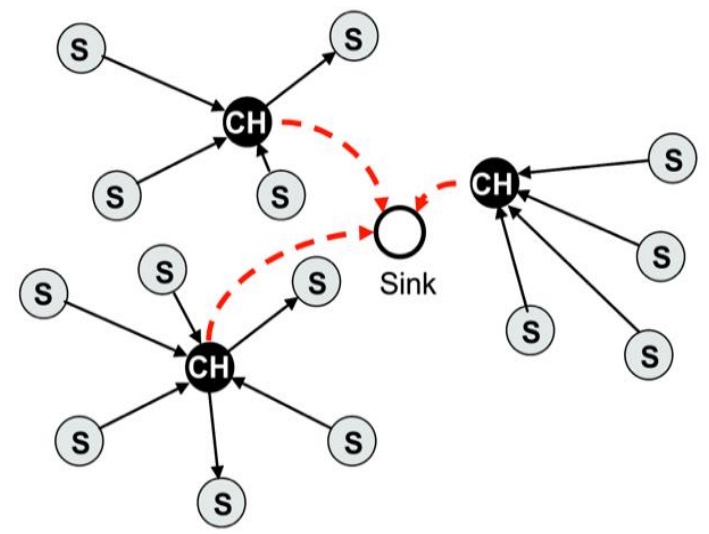

Fig. 5. LEACH protocol

energy considered election mechanism improves the lifetime over LEACH. An improved algorithm DWEHC (Distributed Weight-based Energy-efficient Hierarchical Clustering protocol) is proposed by Gupta in [12]. Like HEED, the residual energy is considered in DWEHC with the cluster range for calculating the weight of each node. Within cluster, the no-leader nodes use the knowledge of distance to decide to be a single-level or multi-level member. This location awareness balances the cluster size and improves the energy efficiency.

\subsection{Tree-based architecture}

Another widely used approach in WSN is the tree-based architecture, where the sensors nodes transmit data following a tree structure network via intermediate nodes to the sink. Processing operations such as data aggregation can be performed at intermediate nodes to reduce the data transmission and save energy.

An Energy-Aware Distributed Aggregation Tree (EADAT) is proposed in [13]. This approach takes the residual energy and the number of hops to the sink into account to construct the tree. A control message which contains five fields (ID, parent, power, status, hop Cnt) is defined for the construction. Where ID and parent records the node's identification in the network, and its parent node respectively; power represents the node residual power; status indicates the state of the sending node in the network (undefined state, leaf node, non-leaf node, or danger state); hopCnt is the number of hops to reach the sink. During its construction process, the sink broadcasts initial control message, the node $v$ chooses a neighbor with the higher residual power and shortest path to the sink as its parent. It then broadcasts a message with its own hopCnt. Another node $u$ will make itself as a non-leaf node if it receives a message from $v$ with parent $=u$. Otherwise, it will be a leaf node of $v$. Once all nodes broadcast their message and find its status in the network, the construction of tree is completed. 
A Power Efficient Data gathering and Aggregation Protocol (PEDAP) was proposed by Tan in [14]. It is a near optimal minimum spanning tree-based (MST) protocol. PEDAP aims to improve the lifetime by minimizing the total energy consumption in communication for each round. In this approach, the computation of the routing tree is completed in a centralized manner at the BS. A Prim's MST algorithm that is a greedy algorithm for constructing MST with the minimum edge weight is used for the computation. MST example is shown in Fig. 6. MST ensures a minimal weight route for the whole network; However, the running time complexity is $O\left(n^{2}\right)$ where $n$ is the number of nodes in the network. Compared with LEACH and PEGASIS, the authors prove that PEDAP and PEADP-PA significantly improve the time of the first dead node.

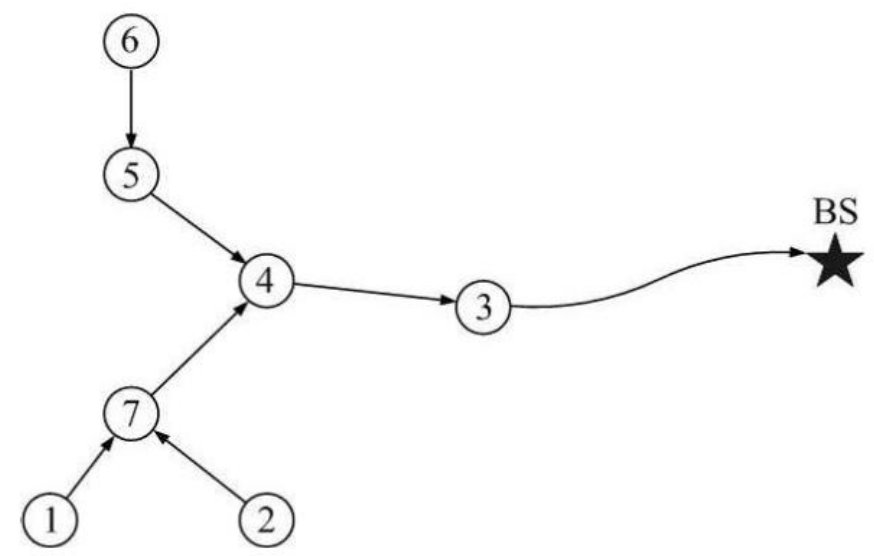

Fig. 6. Minimum spanning tree-based routing protocol

The Tiny AGgregation (TAG) approach is a data-centric tree-based protocol [15]. A declarative interface is used for data collection and aggregation. With a given query, only the relevant information is sent to the sink periodically. This algorithm has two phases: the distributed phase and the collection phase. The construction of tree is established in the first phase. The sink first broadcasts a message which contains the distance to the sink. When a node (not already in the tree) receives a message, it sets the sending node as its parent. The node then broadcasts again the same message with its own ID and distance. After the tree is constructed, a semantic query which specifies the required type of data (such as temperature or humidity) will be sent by the sink to the network following the tree. This data-centric tree-based algorithm reduces the number of involved nodes among the network and improves the network lifetime. However, it is not robust to link failure and the re-organization is highly energy consuming.

\section{$5 \quad$ Synthesis}

The different approaches presented above are synthetized in Table 1. For our application purposed, different performance metrics for the data aggregation routing protocols 
have been chosen and are described as follows: energy efficiency represents the algorithm's efficiency for data collection; Maintaining cost represents the energy consumption for reconstruction of the architecture; Resilience to link failure indicates the robustness of the network facing the loss of link when a node dies; Scalability stands for the capability to scale the scenario; The plus and minus are used to represent the strength and weakness of these routing protocols. This table can then be used to select suitable algorithms for our application case.

Table 1. SUMMARY OF DATA AGGREGATION PROTOCOLS IN WSN

\begin{tabular}{|c|c|c|c|c|}
\hline $\begin{array}{c}\text { Protocol name } \\
\text { (Type) }\end{array}$ & $\begin{array}{c}\text { Energy effi- } \\
\text { ciency }\end{array}$ & $\begin{array}{c}\text { Maintaining } \\
\text { cost }\end{array}$ & $\begin{array}{c}\text { Resilience to } \\
\text { link failure }\end{array}$ & Scalability \\
\hline$P E G A S I S(C h)$ & - & -- & - & -- \\
\hline$C C M(C h)$ & + & - & - & -- \\
\hline$C R B C C(C h)$ & + & + & + & - \\
\hline$B C B R P(C h)$ & - & -- & ++ & -- \\
\hline$L E A C H(C l)$ & -- & + & + & -- \\
\hline$T L-L E A C H(C l)$ & - & + & + & + \\
\hline$H E E D(C l)$ & + & - & + & + \\
\hline$D W E H C(C l)$ & ++ & - & ++ & + \\
\hline$E A D A T(T r)$ & - & -- & - & + \\
\hline$P E D A P(T r)$ & ++ & + & - & + \\
\hline$T A G(T r)$ & + & ++ & - & + \\
\hline
\end{tabular}

Due to the difference of the communicating concretes behaviors over the three phases, we aim to find the most suitable approach for each phase. As mentioned above, the communicating concrete mainly monitors its status during the manufacturing. Therefore, a simple and efficient data aggregation protocol will be preferred. We can see from the table that DWEHC and PEDAP have better energy efficiency than the others. Meanwhile, DWEHC provides a high resilience to link failures, PEDAP has a low maintaining cost. In this case, both are considered relevant for the manufacturing phase.

For construction, the problems are not only the minimization of energy inside each beam, but also the re-organization of network between concrete beams. Thus, a data aggregation technique favoring dynamic and low maintaining cost network should be privileged. Although the cluster-based approach like DWEHC provides a good scalability and high energy efficiency, its high maintaining costs are not suitable for this phase. In contrast, PEDAP has a low maintaining cost with a good scalability and high energy efficiency and thus may be a good choice for this phase. Although TAG provides a lower energy efficiency than PEDAP, its scalability and lower maintaining cost may also give a good result.

After the construction, a large network will be completed and used for the exploitation phase, a robust and low maintaining cost protocol will be adapted. Therefore, a 
protocol with high energy efficiency, high tolerance to link failure and low maintaining cost will be the best choice. From the table we can see that CRBCC may have good result, but it cannot be used in the context of communicating material, because it needs that all the nodes can directly communicate with the BS. The high energy efficiency and resilience to link failure of DWEHC may lead a good result for periodical monitoring. In addition, different physical phenomena will be monitored, such as temperature, humidity, etc. The data-centric tree-based routing protocol TAG will also be considered.

\section{Conclusion and future work}

In this paper, the concept of communicating concrete is introduced in the framework of the McBIM project. Holonic manufacturing approach is presented with four main challenges (two for Physical part and two for Digital part). The existing solutions of data aggregation architectures for the Physical part are discussed with its advantages and drawbacks. Finally, those algorithms are compared with the required performance metrics for application on the McBIM project.

The future work will aim to test and analyze the energy consumption of the data aggregation algorithms that would have been previously selected. These results will confirm if the aggregation approach could significantly improve the lifetime of the WSN for the physical part of our approach. After the physical processing part is completed, we will then continue the development of the digital part, construct its dedicated multi-agent systems and specify the hierarchical and heterarchical relationships. Furthermore, these data will be interpreted via BIM standard for manufacturers, builders and operators. The use of communicating concrete not only reduces the inventory for all operators, but also provides a strong support for building health monitoring.

\section{References}

1. S. Kubler, K. Främling, W. Derigent, and M. J. Rabita, "Premiers travaux relatifs au concept de matière communicante: Processus de dissémination des informations relatives au produit," Thèse de doctorat, Université de Lorraine, 2012.

2. K. Mekki, "Gestion de l'information embarquée dans des matériaux communicants à l'aide de protocoles de réseaux de capteurs," Thèse de doctorat, Université de Lorraine, 2016.

3. "ANR Project McBIM," 2017. [Online]. Available: http://mcbim.cran.univ-lorraine.fr/Members.

4. H. Van Brussel, J. Wyns, P. Valckenaers, L. Bongaerts, and P. Peeters, "Reference Architecture for Holonic Manufacturing Systems," Comput. Ind., vol. 37, no. 3, pp. 255-274, 1998.

5. E. Fasolo, M. Rossi, J. Widmer, and M. Zorzi, "in-Network Aggregation Techniques for Wireless Sensor Networks: a Survey,” IEEE Wirel. Commun., no. April, pp. 70-87, 2007.

6. S. Lindsey, C. Raghavendra, and K. M. Sivalingam, "Data gathering algorithms in sensor networks using energy metrics," IEEE Trans. Parallel Distrib. Syst., vol. 13, no. 9, pp. 924935, 2002. 
7. F. Tang, I. You, S. Guo, M. Guo, and Y. Ma, "A chain-cluster based routing algorithm for wireless sensor networks,” J. Intell. Manuf., vol. 23, no. 4, pp. 1305-1313, 2012.

8. G. Zheng and Z. Hu, "Chain Routing Based on Coordinates-Oriented Clustering Strategy in WSNs," Int. Symp. Comput. Netw. Multimed. Technol., vol. 2, no. 1, pp. 1-4, 2010.

9. K. S. Ahn, D. G. Kim, B. S. Sim, H. Y. Youn, and O. Song, "Balanced chain-based routing protocol (BCBRP) for energy efficient wireless sensor networks," Proc. - 9th IEEE Int. Symp. Parallel Distrib. Process. with Appl. Work., pp. 227-231, 2011.

10. W. R. Heinzelman, A. Chandrakasan, and H. Balakrishnan, "Energy-efficient communication protocol for wireless microsensor networks," Proc. 33rd Annu. Hawaii Int. Conf. Syst. Sci., vol. 8, p. 8020, 2000.

11. O. Younis and S. Fahmy, "HEED: A hybrid, energy-efficient, distributed clustering approach for ad hoc sensor networks," IEEE Trans. Mob. Comput., vol. 3, no. 4, pp. 366-379, 2004.

12. P. Ding, J. Holliday, and A. Celik, "Distributed Energy-Efficient Hierarchical Clustering for Wireless Sensor Networks," IEEE Trans. Commun., pp. 322-339, 2005.

13. M. Ding, X. Cheng, and G. Xue, "Aggregation tree construction in sensor networks," IEEE 58th VTC, p. 2168-2172 Vol.4, 2004.

14. H. Ö. Tan and I. Körpeoğlu, "Power efficient data gathering and aggregation in wireless sensor networks," ACM SIGMOD Rec., vol. 32, no. 4, p. 66, 2005.

15. S. Madden, M. J. Franklin, J. M. Hellerstein, and W. Hong, "TAG: A Tiny AGgregation Service for Ad-hoc Sensor Networks,” Osdi, vol. 36, no. SI, pp. 131-146, 2002. 